\title{
Plasma levels of serum amyloid A1 and mortality after exposure to high-dose radiation
}

\author{
Dmitry Klokov ${ }^{1,2}$
}

${ }^{1}$ Laboratory of Radiobiology and Radiotoxicology, Department of Research on Biological and Health Effects of Ionizing Radiation, Institute of Radioprotection and Nuclear Safety (IRSN), Fontenay-aux-Roses, France; ${ }^{2}$ Department of Biochemistry, Microbiology and Immunology, University of Ottawa, Ottawa, Ontario, Canada

Correspondence to: Dmitry Klokov. Laboratory of Radiobiology and Radiotoxicology, Department of Research on Biological and Health Effects of Ionizing Radiation, Institute of Radioprotection and Nuclear Safety (IRSN), IRSN/PSE-SANTE/SESANE/LRTOX, Bt. 27, 92262 Fontenay-auxRoses Cedex, France. Email: dmitry.klokov@irsn.fr.

Provenance and Peer Review: This article was commissioned by the editorial office, Annals of Translational Medicine. The article did not undergo external peer review.

Comment on: Huang J, Qi Z, Chen M, et al. Serum amyloid A1 as a biomarker for radiation dose estimation and lethality prediction in irradiated mouse. Ann Transl Med 2019;7:715.

Submitted Mar 02, 2020. Accepted for publication Mar 29, 2020.

doi: 10.21037/atm.2020.03.172

View this article at: http://dx.doi.org/10.21037/atm.2020.03.172

Presented in Annals of Translational Medicine, Jinfeng Huang and colleagues' study (1) of the association between tissue/ plasma levels of the serum amyloid A1 (SAA1) protein and dose of acute ionizing radiation exposure, highlights the potential benefits of SAA1 monitoring for triage purposes during potential mass-casualty events of exposure of humans to unknown doses of ionizing radiation. Two relatively separate potential utilities of measuring SAA1 in high-dose radiation exposed humans are proposed and discussed by the authors: one is reconstruction of exposure dose and second is prediction of adverse health outcome.

Scenarios of potential undesired human exposure to high doses of ionizing radiation ( $>1$ Gy) include nuclear power plant accidents, such as Chernobyl and Fukushima (2), dirty bomb terrorist attacks (3) and military activities and warfare using nuclear weapons (4). Although the scenarios of nuclear power plant accidents have been of primary concern for decades, the terrorist and military nuclear events have gained attention due to the recent political insurgencies worldwide and, most recently, alleged changes in the US and Russia's national military postures implying the use of tactical nuclear weapons in regional conflicts (5). It is important to note that undesired exposures of humans to high-dose radiation are extremely rare. Even during nuclear accidents, the vast majority of exposed individuals receive low to moderate doses of radiation that do not cause life threatening acute disease. Nonetheless, identifying highdose exposed individuals in a timely fashion would save lives; therefore, preparedness for triage of irradiated human cohorts is an important issue.

Such triage would involve medical surveillance and various tests to reconstruct radiation exposure dose to segregate exposed groups for prioritization of remediation and treatment $(6,7)$. Dose reconstruction using biological end-points is collectively referred to as biodosimetry. Currently, the "golden standard" for human biodosimetry is the assessment of the frequency of dicentric chromosome aberrations in peripheral blood lymphocytes or the dicentric chromosome assay (DCA) (8). This analysis is however prone to large variability due to several factors, such as the need for laboratory manipulations to mitotically stimulate lymphocytes and thus producing variable outcomes or human subjectivity in scoring dicentric chromosome aberrations. Therefore, in order to maintain emergency preparedness, countries maintain their national biodosimetry facilities and capabilities and regularly perform emergency exercises, as wells as intercomparison studies involving multiple countries and aiming at standardization of the DCA $(9,10)$. Although various automation and optimization solutions for performing the DCA have been proposed $(11,12)$, no consensus exist and the assay is still widely considered a labor-intensive and time-consuming 


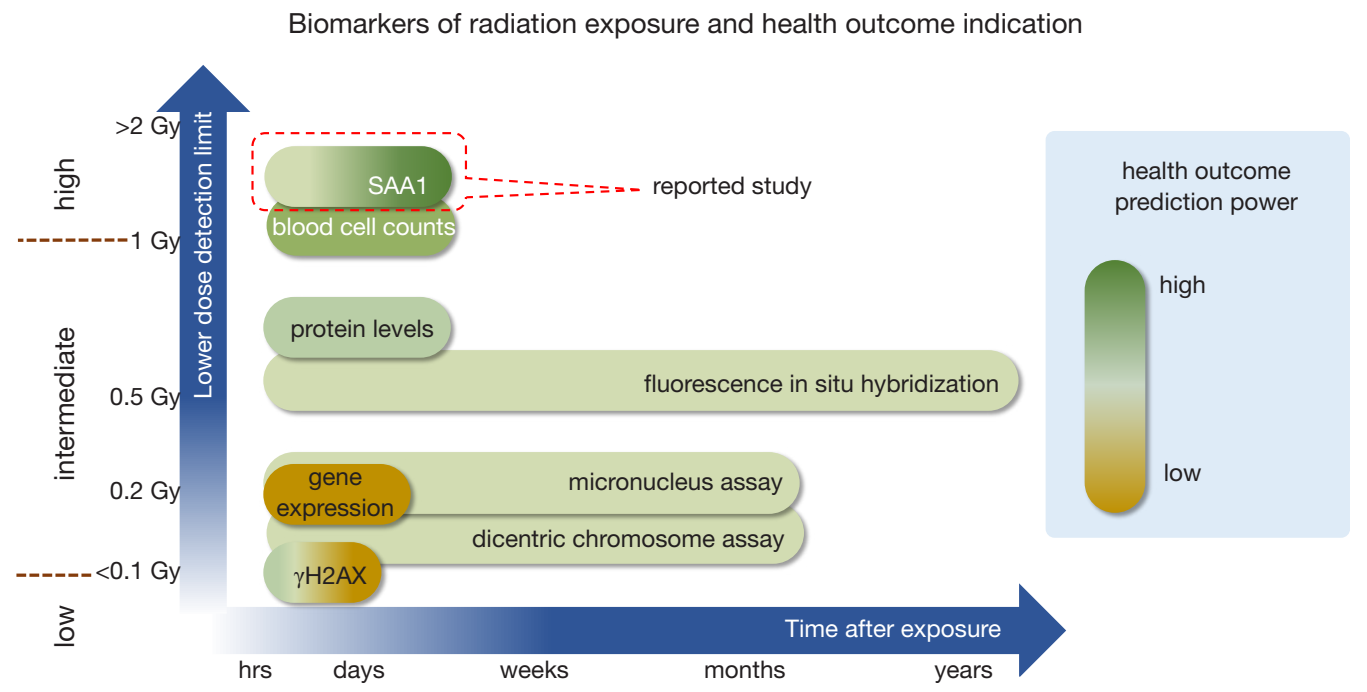

Figure 1 A diagram showing a relationship between the lower dose detection limit, the applicability time period after exposure and the health outcome prediction power for several biomarkers/assays for potential use in biodosimetry and health management upon accidental exposure of humans to unknown doses of ionizing radiation. The results by Huang et al. (1) (red dashed selection) are strongly relevant to the ability to predict adverse health outcome.

technique presenting a bottleneck for an adequate response to a potential mass-casualty event. Alternative methods for reconstructing unknown radiation dose have been explored (13), including those based on a widely known marker of DNA double-strand breaks $\gamma \mathrm{H} 2 \mathrm{AX}$ (14), gene expression changes (14) and several other biomarkers of exposure to radiation. High-throughput automation have also been proposed for non-dicentric based methods (15).

The idea of measuring blood plasma levels of various proteins for biodosimetry and triage is an attractive alternative to the DCA since results can be obtained much faster. Also, this work does not involve sophisticated microscopy analyses and is adaptable for high-throughput automation. Sure enough, such studies have been carried out previously, however blood plasma proteins turned out to be of limited use at lower doses. Indeed, the lower dose detection limit of the DCA of 0.2 Gy could not be reached using blood plasma proteins $(16,17)$ (Figure 1). It is not surprising then that Huang et al. could only see SAA1 increases in the plasma of mice exposed to doses of $\geq 2$ Gy, suggesting that it can be used for exclusively highdose exposed individuals and should be combined with other more sensitive techniques. Arguably, this represents a limitation of the utility of SAA1 as a biomarker of exposure dose since, as was mentioned before, majority of exposed people upon mass-casualty nuclear accidents would be exposed to low doses.

Why the choice of the authors fell onto the SAA1 protein? It is a member of a small family of serum amyloid A proteins (SAA). SAA1 constitutes about $70 \%$ of acutephase SAA response to proinflammatory stimuli (18) and has been implicated in a wide range of human pathologies, including respiratory disease, and it was proposed as a prognostic marker (19). Since acute radiation syndrome upon high-dose exposure is accompanied by acute systemic inflammation, the choice of Huang et al. to examine the link of SAA1 with acute radiation exposure outcomes was logical and well justified. Indeed, SAA has previously been implicated in acute radiation toxicity and proposed, in combination with other protein markers and blood cell counts, as a biomarker of exposure $(17,20)$. Several aspects pursued by Huang et al. in their study allowed the authors to generate important novel knowledge.

Firstly, they examined the plasma levels of SAA1 in the extended period of time post-irradiation, which resulted in a very interesting bi-phasic kinetics of SAA1 increases. The initial peak between 6-24 h disappeared and reappeared at $>5$ days post-exposure in high-dose exposed mice only. One potential implication of this discovery is the possibility to perform this assay days after exposure of humans, when earlier screening could not be done, to identify severely injured victims. 
Second important observation was that SAA1 levels responded not only to total-body irradiation, but also to various partial-body irradiation modes that either included or excluded the liver, a major producer of systemic SAA1. Upon real-life exposure scenarios, it is anticipated that various body parts of humans may be exposed to radiation differently. The ability of the plasma SAA1 levels to respond to partial body exposures suggests its utility for such complex exposure patterns. However, it is important to note that under such circumstances, the SAA1 plasma level would rather serve as a biomarker of health effect, but not exposure dose.

Last, and arguably most important result of the study, is the revealed correlation between the second peak of SAA1 and the mortality of the exposed mice. Huang and colleagues are to be applauded for noticing and exploring in detail the inverse correlation between the SAA1 levels at 5 and 7 days post-exposure and the survivability of animals. They carried out an additional experiment in which they monitored SAA1 levels longitudinally in 10 Gy exposed mice and, using individual mouse data, estimated specificity, sensitivity and precision of predicting lethality by measured SAA1 levels. They show that using a cut-off value of $1.15 \mu \mathrm{g} / \mathrm{mL}$ of SAA1 on day 7 post-exposure, a specificity of $2 / 3$, sensitivity of $13 / 16$ and precision of $13 / 14$ could be achieved for predicting a lethal outcome. The authors further validate their result by protecting lethally irradiated mice with a radioprotecting drug amifostine and demonstrating that such protection was accompanied by the reduction of SAA1 plasma levels when compared to the radiation only group. Curiously, a month after the publication by Huang et al., an independent report was published showing that in mice rescued from the acute radiation syndrome by a drug captopril, the improvement correlated with reduced plasma levels of SAA1 (21), consistent with the major observation of the study by Huang and colleagues.

Can these results obtained in mice be extrapolated to humans? Inability to reproduce effects observed in preclinical studies in humans is a well appreciated issue in translational biomedical research (22-24). Unfortunately, biodosimetry research area may also be flawed by inappropriate mouse-to-human extrapolation. Thus, it was shown that many radiation-responsive genes changed in opposite directionalities in human and mouse cells (25). To this end, the final piece of data presented by Huang and colleagues is very important. They validated their mouse results in a cohort of human nasopharyngeal carcinoma patients, showing that blood plasma SAA1 concentration increased after local irradiation of the diseased tissue at 60-70 Gy.

To truly improve the knowledge on the SAA1 utility for biomonitoring victims of radiological accidents, further research will require a large human patient cohort size, more mechanistic data relating SAA1 response to specific pathological conditions, and validation of the results for mixed radiation exposure, e.g., gamma-radiation + neutrons, that are typical for real-life scenarios. This reported study nonetheless represents an important step forward in managing severe acute radiation syndrome in accidentally exposed human cohorts.

\section{Acknowledgments}

Dr. Dmitry Klokov is supported by the Health and Environment Research Program of the Institute of Radioprotection and Nuclear Safety (IRSN), France.

Funding: None.

\section{Footnote}

Conflicts of Interest: The author has completed the ICMJE uniform disclosure form (available at http://dx.doi. org/10.21037/atm.2020.03.172). The author has no conflicts of interest to declare.

Ethical Statement: The author is accountable for all aspects of the work in ensuring that questions related to the accuracy or integrity of any part of the work are appropriately investigated and resolved.

Open Access Statement: This is an Open Access article distributed in accordance with the Creative Commons Attribution-NonCommercial-NoDerivs 4.0 International License (CC BY-NC-ND 4.0), which permits the noncommercial replication and distribution of the article with the strict proviso that no changes or edits are made and the original work is properly cited (including links to both the formal publication through the relevant DOI and the license). See: https://creativecommons.org/licenses/by-nc-nd/4.0/.

\section{References}

1. Huang J, Qi Z, Chen M, et al. Serum amyloid A1 as a biomarker for radiation dose estimation and lethality prediction in irradiated mouse. Ann Transl Med 
2019;7:715.

2. Dallas CE. Medical lessons learned from chernobyl relative to nuclear detonations and failed nuclear reactors. Disaster Med Public Health Prep 2012;6:330-4.

3. Goffman T. Lack of strategic insight: the "dirty bomb" effort. Am J Disaster Med 2009;4:181-3.

4. Reeves GI. Medical implications of enhanced radiation weapons. Mil Med 2010;175:964-70.

5. Woolf AF. Russia's Nuclear Weapons: Doctrine, Forces, and Modernization. In: Congressional Research Service Report for Congress, 2020:14.

6. Jaworska A, Ainsbury EA, Fattibene P, et al. Operational guidance for radiation emergency response organisations in Europe for using biodosimetric tools developed in EU MULTIBIODOSE project. Radiat Prot Dosimetry 2015;164:165-9.

7. Rojas-Palma C, Liland A, Jerstad AN, et al. TMT Handbook. Triage, monitoring and treatment of people exposed to ionising radiation following a malevolent act. Norway: NRPA, 2009.

8. World Health Organization. Cytogenetic dosimetry: applications in preparedness for and response to radiation emergencies. Vienna: International Atomic Energy Agency, 2011.

9. Oestreicher U, Samaga D, Ainsbury E, et al. RENEB intercomparisons applying the conventional Dicentric Chromosome Assay (DCA). Int J Radiat Biol 2017;93:20-9.

10. Wilkins RC, Beaton-Green LA, Lachapelle S, et al. Evaluation of the annual Canadian biodosimetry network intercomparisons. Int J Radiat Biol 2015;91:443-51.

11. Royba E, Repin M, Pampou S, et al. RABiT-II-DCA: a fully-automated dicentric chromosome assay in multiwell plates. Radiat Res 2019;192:311-23.

12. Farkas G, Kocsis ZS, Székely G, et al. Quantifying biological effects of radiation from high-energy linear accelerators on lymphocytes. J Radiat Res 2020;61:73-81.

13. Ainsbury E, Badie C, Barnard S, et al. Integration of new biological and physical retrospective dosimetry methods into EU emergency response plans - joint RENEB and EURADOS inter-laboratory comparisons. Int J Radiat Biol 2017;93:99-109.

14. Andrievski A, Wilkins RC. The response of gamma-H2AX in human lymphocytes and lymphocytes subsets measured in whole blood cultures. Int J Radiat Biol 2009;85:369-76.

15. Garty G, Turner HC, Salerno A, et al. THE DECADE OF THE RABiT (2005-15). Radiat Prot Dosimetry 2016;172:201-6.

16. Göransson Nyberg A, Stricklin D, Sellström Å. Mass casualties and health care following the release of toxic chemicals or radioactive material--contribution of modern biotechnology. Int J Environ Res Public Health 2011;8:4521-49.

17. Ossetrova NI, Blakely WF. Multiple blood-proteins approach for early-response exposure assessment using an in vivo murine radiation model. Int J Radiat Biol 2009;85:837-50.

18. Jensen LE, Whitehead AS. Regulation of serum amyloid A protein expression during the acute-phase response. Biochem J 1998;334:489-503.

19. Vietri L, Fui A, Bergantini L, et al. Serum amyloid A: a potential biomarker of lung disorders. Respir Investig 2020;58:21-7.

20. Ossetrova NI, Condliffe DP, Ney PH, et al. Earlyresponse biomarkers for assessment of radiation exposure in a mouse total-body irradiation model. Health Phys 2014;106:772-86.

21. McCart EA, Lee YH, Jha J, et al. Delayed captopril administration mitigates hematopoietic injury in a murine model of total body irradiation. Sci Rep 2019;9:2198.

22. Peterson JK, Houghton PJ. Integrating pharmacology and in vivo cancer models in preclinical and clinical drug development. Eur J Cancer 2004;40:837-44.

23. Jameson SC, Masopust D. What is the predictive value of animal models for vaccine efficacy in humans? Reevaluating the potential of mouse models for the human immune system. Cold Spring Harb Perspect Biol 2018. doi: 10.1101/cshperspect.a029132.

24. Shanks N, Greek R, Greek J. Are animal models predictive for humans? Philos Ethics Humanit Med 2009;4:2.

25. Ghandhi SA, Smilenov L, Shuryak I, et al. Discordant gene responses to radiation in humans and mice and the role of hematopoietically humanized mice in the search for radiation biomarkers. Sci Rep 2019;9:19434.
Cite this article as: Klokov D. Plasma levels of serum amyloid A1 and mortality after exposure to high-dose radiation. Ann Transl Med 2020;8(11):661. doi: 10.21037/atm.2020.03.172 